\title{
Analysis of Non-Linear Recurrence Relations for the Recurrence Coefficients of Generalized Charlier Polynomials
}

Walter VAN ASSCHE ${ }^{\dagger}$ and Mama FOUPOUAGNIGNI

† Katholieke Universiteit Leuven, Department of Mathematics, Celestijnenlaan 200 B, B-3001 Leuven, Belgium

E-mail:walter@wis.kuleuven.ac.be

$¥$ University of Yaounde, Advanced School of Education, Department of Mathematics, P.O. Box 47, Yaounde, Cameroon

E-mail: foumama@yahoo.fr

This paper is part of the Proceedings of SIDE V;

Giens, June 21-26, 2002

\begin{abstract}
The recurrence coefficients of generalized Charlier polynomials satisfy a system of nonlinear recurrence relations. We simplify the recurrence relations, show that they are related to certain discrete Painlevé equations, and analyze the asymptotic behaviour.
\end{abstract}

\section{Introduction}

Orthogonal polynomials on the real line always satisfy a three-term recurrence relation. For monic polynomials $P_{n}$ this is of the form

$$
P_{n+1}(x)=\left(x-\beta_{n}\right) P_{n}(x)-\gamma_{n} P_{n-1}(x),
$$

with initial values $P_{0}=1$ and $P_{-1}=0$. For the orthonormal polynomials $p_{n}$ the recurrence relation is

$$
x p_{n}(x)=a_{n+1} p_{n+1}(x)+b_{n} p_{n}(x)+a_{n} p_{n-1}(x),
$$

where $a_{n}^{2}=\gamma_{n}$ and $b_{n}=\beta_{n}$. The recurrence coefficients are given by the integrals

$$
a_{n}=\int x p_{n}(x) p_{n-1}(x) d \mu(x), \quad b_{n}=\int x p_{n}^{2}(x) d \mu(x)
$$

and can also be expressed in terms of determinants containing the moments of the orthogonality measure $\mu$ [11]. For classical orthogonal polynomials one knows these recurrence coefficients explicitly, but when one uses non-classical weights, then often one does not 
know the recurrence coefficients explicitly. When the weight satisfies a first order differential equation with polynomial coefficients, then one can use a technique developped by Laguerre, Shohat and Freud $[1,4]$ to obtain non-linear recurrence relations for the recurrence coefficients. The most famous example is the Freud weight $w(x)=\exp \left(-x^{4}\right)$ on the real line $[4,9]$, for which $\beta_{n}=0$ and $\gamma_{n}$ satisfies

$$
\gamma_{n+1}+\gamma_{n}+\gamma_{n-1}=\frac{n}{4 \gamma_{n}}
$$

which is a special case of the discrete Painlevé equation $\mathrm{d}_{-} \mathrm{P}_{\mathrm{I}}[11]$. In this paper we will investigate similar non-linear recurrence relations for discrete orthogonal polynomials which are non-classical extensions of the Charlier polynomials.

\section{Generalized Charlier polynomials}

Charlier polynomials $[2,12]$ are the orthogonal polynomials with respect to the Poisson distribution on the non-negative integers:

$$
w_{k}=\frac{a^{k}}{k !}, \quad k=0,1,2, \ldots
$$

with $a>0$. They are usually denoted by $C_{n}(x ; a)$ and the orthogonality conditions are

$$
\sum_{k=0}^{\infty} C_{n}(k ; a) C_{m}(k ; a) \frac{a^{k}}{k !}=n ! a^{-n} e^{a} \delta_{m, n} .
$$

The three-term recurrence relation for these polynomials is

$$
-x C_{n}(x ; a)=a C_{n+1}(x ; a)-(n+a) C_{n}(x ; a)+n C_{n-1}(x ; a) .
$$

For the monic polynomials $P_{n}(x)=(-a)^{n} C_{n}(x ; a)$ the three-term recurrence relation is

$$
x P_{n}(x)=P_{n+1}(x)+(n+a) P_{n}(x)+a n P_{n-1}(x),
$$

so that

$$
\beta_{n}=n+a, \quad \gamma_{n}=\text { an } .
$$

Generalized Charlier polynomials were introduced in [6]. These are discrete orthogonal polynomials on $\mathbb{N}$ with weights

$$
w_{k}=\frac{a^{k}}{(k !)^{N}}, \quad k=0,1,2 \ldots,
$$

where $N \geq 1$ and $a>0$. For $N=1$ one deals with the ordinary Charlier polynomials. For $N=2$ the recurrence coefficients $\beta_{k}, \gamma_{k+1}(k=0,1,2, \ldots)$ satisfy the Laguerre-Freud equations

$$
\begin{aligned}
\gamma_{n}+\gamma_{n+1} & =-\left(\begin{array}{c}
n \\
2
\end{array}\right)-\beta_{n}^{2}+n \beta_{n}+\sum_{i=0}^{n-1} \beta_{i}+a \\
\left(\beta_{n}+\beta_{n+1}\right) \gamma_{n+1} & =-n \sum_{i=0}^{n} \beta_{i}+n \gamma_{n+1}+\left(\begin{array}{c}
n+1 \\
3
\end{array}\right)+\sum_{i=0}^{n} \beta_{i}^{2}+2 \sum_{i=1}^{n} \gamma_{i}+\gamma_{n+1}
\end{aligned}
$$


with initial values

$$
\beta_{0}=\frac{\sqrt{a} I_{1}(2 \sqrt{a})}{I_{0}(2 \sqrt{a})}, \quad \gamma_{0}=0
$$

where $I_{0}$ and $I_{1}$ are the modified Bessel functions of order 0 and 1 [6].

We will now simplify this system of recurrence relations:

Proposition 1. Let $\beta_{n}=n+b_{n}$, then the recurrence coefficients for generalized Charlier polynomials (for $N=2$ ) satisfy

$$
\begin{aligned}
\left(n+b_{n}+b_{n-1}\right) \gamma_{n} & =n a \\
n a\left(b_{n-1}-b_{n}\right) & =\gamma_{n}\left(\gamma_{n+1}-\gamma_{n-1}\right),
\end{aligned}
$$

with initial conditions

$$
b_{0}=\frac{\sqrt{a} I_{1}(2 \sqrt{a})}{I_{0}(2 \sqrt{a})}, \quad \gamma_{0}=0, \gamma_{1}=a-b_{0}^{2} .
$$

Proof. If we put $\beta_{n}=n+b_{n}$, then we have

$$
\sum_{i=0}^{n-1} \beta_{i}=\left(\begin{array}{l}
n \\
2
\end{array}\right)+\sum_{i=0}^{n-1} b_{i}, \quad \sum_{i=0}^{n} \beta_{i}^{2}=\sum_{i=0}^{n} b_{i}^{2}+2 \sum_{i=1}^{n} i b_{i}+\frac{n^{3}}{3}+\frac{n^{2}}{2}+\frac{n}{6} .
$$

If we insert this into (2.1), then this gives

$$
\gamma_{n+1}+\gamma_{n}=\sum_{i=0}^{n-1} b_{i}-b_{n}^{2}-n b_{n}+a,
$$

and (2.2) becomes

$$
\left(2 n+1+b_{n}+b_{n+1}\right) \gamma_{n+1}=(n+1) \gamma_{n+1}-n \sum_{i=0}^{n-1} b_{i}+n b_{n}+2 \sum_{i=1}^{n-1} i b_{i}+\sum_{i=0}^{n} b_{i}^{2}+2 \sum_{i=1}^{n} \gamma_{i} .
$$

Summing both sides of (2.6) gives

$$
\gamma_{n+1}+2 \sum_{i=1}^{n} \gamma_{i}=-2 \sum_{i=1}^{n-1} i b_{i}-n b_{n}-\sum_{i=0}^{n} b_{i}^{2}+n \sum_{i=0}^{n-1} b_{i}+(n+1) a,
$$

and if we use this in (2.7) then we find (2.3). Next, we apply the difference operator to both sides of (2.6) to find

$$
\gamma_{n+1}-\gamma_{n-1}=\left(b_{n-1}-b_{n}\right)\left(n+b_{n}+b_{n-1}\right) \text {. }
$$

If we now use (2.3), then this gives (2.4).

The equations can be put into a somewhat more pleasing form, involving a special case of the discrete Painlevé II equation. 
Proposition 2. For every $n \geq 1$ we have $0<\gamma_{n}<a$ and $b_{n}>0$. If we put $\gamma_{n}=a\left(1-c_{n}^{2}\right)$ then (2.3)-(2.4) can be written as

$$
\begin{gathered}
b_{n}=\sqrt{a} c_{n} c_{n+1} \\
n c_{n}=\sqrt{a}\left(c_{n+1}+c_{n-1}\right)\left(1-c_{n}^{2}\right), \\
\text { with } c_{1}=I_{1}(2 \sqrt{a}) / I_{0}(2 \sqrt{a}) \text { and } c_{0}=1 .
\end{gathered}
$$

Proof. Since $\gamma_{n}$ are recurrence coefficients of orthogonal polynomials, it follows that $\gamma_{n}>$ 0 for $n \geq 1$. Summing (2.4) gives

$$
a\left(\sum_{k=0}^{n-1} b_{k}-n b_{n}\right)=\gamma_{n} \gamma_{n+1},
$$

but on the other hand (2.6) gives

$$
\sum_{k=0}^{n-1} b_{k}-n b_{n}=\gamma_{n+1}+\gamma_{n}+b_{n}^{2}-a .
$$

Combining both results gives

$$
a\left(\gamma_{n+1}+\gamma_{n}+b_{n}^{2}-a\right)=\gamma_{n} \gamma_{n+1} .
$$

This can be written as

$$
\left(\gamma_{n}-a\right)\left(\gamma_{n+1}-a\right)=a b_{n}^{2}
$$

hence $\gamma_{n}-a$ and $\gamma_{n+1}-a$ have the same sign. The initial condition $\gamma_{1}=a-b_{0}^{2}$ hence implies that $\gamma_{n}-a<0$ for every $n \geq 1$. Therefore we can write $\gamma_{n}=a-a c_{n}^{2}$ and we find (2.10). If we insert this into (2.3), then we find

$$
a\left(1-c_{n}^{2}\right)\left(n+\sqrt{a} c_{n} c_{n+1}+\sqrt{a} c_{n} c_{n-1}\right)=n a,
$$

which can easily be reduced to (2.11).

Recall that the discrete Painlevé II equation d- $\mathrm{P}_{\mathrm{II}}$ is given by [5]

$$
x_{n+1}+x_{n-1}=\frac{x_{n} z_{n}+\gamma}{1-x_{n}^{2}},
$$

where $z_{n}=\alpha n+\beta$. The equation (2.11) for $c_{n}$ is of this form with $\beta=\gamma=0$ and $\alpha=1 / \sqrt{a}$.

We now have the following asymptotic behaviour of the recurrence coefficients.

Theorem 1. For the recurrence coefficients $\left(\beta_{n}, \gamma_{n}\right)$ of generalized Charlier polynomials we set $\beta_{n}=n+b_{n}$. Then

$$
\lim _{n \rightarrow \infty} \gamma_{n}=a, \quad \lim _{n \rightarrow \infty} b_{n}=0 .
$$

Proof. If we write $\gamma_{n}=a\left(1-c_{n}^{2}\right)$, then $0<\gamma_{n}<a$ implies that $0<c_{n}^{2}<1$ for $n \geq 1$. This mean that the right hand side of (2.11) remains bounded as $n \rightarrow \infty$. But then $n c_{n}$ remains bounded as well, which implies that $c_{n} \rightarrow 0$, and hence $\gamma_{n} \rightarrow a$. The asymptotic behavior of $b_{n}$ then easily follows from (2.10).

The asymptotic behaviour given in the previous theorem was already suggested by the remarks in [6, p. 364]. 


\section{Concluding remarks}

We have found that the recurrence coefficients of generalized Charlier polynomials with $N=2$ are given by the equations (2.10)-(2.11). The original Laguerre-Freud equations (2.1)-(2.2) are more complicated and some work was needed to find the underlying discrete Painlevé equation. It would be more convenient to have a method that gives the simple equations (2.10)-(2.11) directly.

The asymptotic behaviour of the recurrence coefficients allows us to obtain the asymptotic zero distribution of the generalized Charlier polynomials, using the results from [7]. If $x_{1, n}<x_{2, n}<\cdots<x_{n, n}$ are the zeros of $P_{n}$, then the contracted zero distribution

$$
\mu_{n}=\frac{1}{n} \sum_{j=1}^{n} \delta_{x_{j, n} / n}
$$

where $\delta_{c}$ is a Dirac probability measure concentrated at $c$, converges weakly to the uniform distribution on $[0,1]$, just as in the case of the usual Charlier polynomials [7, p. 190]. This result can also be obtained from the behaviour of the orthogonality weights $w(k)=w_{k}=$ $a^{k} /(k !)^{2}$, for which

$$
\log w(k)=k \log a-2 \log k ! \sim k \log a-2\left(k+\frac{1}{2}\right) \log k+2 k .
$$

The growth of this weight is dominated by the term $k \log k$, which grows faster than the rate $k$ at which the points in the support tend to infinity. Following remarks in [8, p. 197], we see that the asymptotic zero distribution is indeed given by the uniform distribution on $[0,1]$, as is the case for the usual Charlier polynomials [8, p. 200]. The asymptotic behaviour of the recurrence coefficients also gives information about the largest zero $x_{n, n}$. In the formula

$$
\beta_{n}=\int x p_{n}^{2}(x) d \mu(x)
$$

we can compute the integral exactly using Gauss quadrature at the $n+1$ zeros of the orthogonal polynomial $P_{n+1}$, so that

$$
\beta_{n}=\sum_{j=1}^{n+1} x_{j, n+1} p_{n}^{2}\left(x_{j, n+1}\right) \lambda_{j, n+1},
$$

where $\lambda_{j, n+1}>0$ are the Christoffel numbers [2]. Since $x_{j, n+1} \leq x_{n+1, n+1}$ for every $j$, we easily find

$$
\beta_{n} \leq x_{n+1} \sum_{j=1}^{n+1} p_{n}^{2}\left(x_{j, n+1}\right) \lambda_{j, n+1}=x_{n+1, n+1},
$$

so that $\beta_{n}$ is a lower bound for the largest zero $x_{n+1, n+1}$. The results in [7] and [8] show that $\lim _{n \rightarrow \infty} x_{n, n} / n=1$.

The recurrence coefficients of generalized Meixner polynomials [13], which are the orthogonal polynomials on the linear lattice with orthogonality weight

$$
w_{k}=\frac{\left(\alpha_{1}\right)_{k}\left(\alpha_{2}\right)_{k}}{(k !)^{2}} c^{k}, \quad k=0,1,2,3, \ldots
$$


with $\alpha_{1}, \alpha_{2}>0$ and $0<c<1$ satisfy recurrence relations [3] which are somewhat similar to $(2.1)-(2.2)$, but now with three parameters $\alpha_{1}, \alpha_{2}$ and $c$ :

$$
\begin{aligned}
(1-c)\left(\gamma_{n}+\gamma_{n+1}\right)= & -(1-c)\left(\begin{array}{c}
n \\
2
\end{array}\right)-(1-c) \beta_{n}^{2}+\left[(1+c) n+c\left(\alpha_{1}+\alpha_{2}\right)\right] \beta_{n} \\
& +(1+c) \sum_{i=0}^{n-1} \beta_{i}+c\left(\alpha_{1}+\alpha_{2}\right) n+c \alpha_{1} \alpha_{2} \\
(1-c)\left(\beta_{n}+\beta_{n+1}\right) \gamma_{n+1}= & -n \sum_{i=0}^{n} \beta_{i}+\left[(1+c) n+c\left(\alpha_{1}+\alpha_{2}\right)+1\right] \gamma_{n+1} \\
& +\left(\begin{array}{c}
n+1 \\
3
\end{array}\right)+\sum_{i=0}^{n} \beta_{i}^{2}+2 \sum_{i=1}^{n} \gamma_{i}
\end{aligned}
$$

with initial values

$$
\beta_{0}=c \alpha_{1} \alpha_{2} \frac{{ }_{2} F_{1}\left(\alpha_{1}+1, \alpha_{2}+1 ; 2 ; c\right)}{{ }_{2} F_{1}\left(\alpha_{1}, \alpha_{2} ; 1 ; c\right)}, \quad \gamma_{0}=0
$$

where ${ }_{2} F_{1}$ is Gauss' hypergeometric function. The analysis of these equations is more involved and will be presented in another paper, where we will show that the asymptotic behaviour conjectured in [14] indeed holds.

\section{Acknowledgements}

This research was supported by INTAS project 00-272 and by FWO research project G.0184.02.

\section{References}

[1] S. Belmehdi, A. Ronveaux, Laguerre-Freud equations for the recurrence coefficients of semiclassical orthogonal polynomials, J. Approx. Theory 76 (1994), 351-368.

[2] T. S. Chihara, An Introduction to Orthogonal Polynomials, Gordon and Breach, New York, 1978.

[3] M. Foupouagnigni, M. N. Hounkonnou, A. Ronveaux, Laguerre-Freud's equations for the recurrence coefficients of $D_{\omega}$-semi-classical orthogonal polynomials of class one, J. Comput. Appl. Math. 99 (1998), 143-154.

[4] G. Freud, On the coefficients in the recursion formulae of the orthogonal polynomials, Proc. Roy. Irish Acad. Sect. A 76 (1976), no. 1, 1-6.

[5] B. Grammaticos, F. W. Nijhoff, A. Ramani, Discrete Painlevé equations, in 'The Painlevé property: one century later' (R. Conte, ed.), CRM Ser. Math. Phys., Springer, New York, 1999, pp. 413-516.

[6] M. N. Hounkonnou, C. Hounga, A. Ronveaux, Discrete semi-classical orthogonal polynomials: Generalized Charlier, J. Comput. Appl. Math. 114 (2000), 361-366. 
[7] A. B. J. Kuijlaars, W. Van Assche, The asymptotic zero distribution of orthogonal polynomials with varying recurrence coefficients, J. Approx. Theory 99 (1999), 167-197.

[8] A. B. J. Kuijlaars, W. Van Assche, Extremal polynomials on discrete sets, Proc. London Math. Soc. (3) 79 (1999), 191-221.

[9] A. P. Magnus, On Freud's equations for exponential weights, J. Approx. Theory 46 (1986), 65-99.

[10] A. P. Magnus, Painlevé-type differential equations for the recurrence coefficients of semiclassical orthogonal polynomials, J. Comput. Appl. Math. 57 (1995), 215-237.

[11] A. P. Magnus, Freud's equations for orthogonal polynomials as discrete Painlevé equations, in 'Symmetries and Integrability of Difference Equations' (Canterbury, 1996), London Math. Soc. Lecture Note Ser. 255, Cambridge Univ. Press, Cambridge, 1999, pp. 228-243.

[12] A. F. Nikiforov, S. K. Suslov, V. B. Uvarov, Classical Orthogonal Polynomials of a Discrete Variable, Springer Series in Computational Physics, Springer-Verlag, Berlin, 1991.

[13] A. Ronveaux, Discrete semi-classical orthogonal polynomials: Generalized Meixner, J. Approx. Theory 46 (1986), 403-407.

[14] A. Ronveaux, Asymptotics for recurrence coefficients in the generalized Meixner case, $J$. Comput. Appl. Math. 133 (2001), 695-696. 